\title{
Hypertrophic Cardiomyopathy Complicated with Acute Myocardial Infarction due to Coronary Embolism
}

\author{
Toru Iwama, Kouichi Asami, Ichirou Kubo and Hidemasa Kitazume
}

\begin{abstract}
We report a case of acute myocardial infarction due to coronary embolism in a patient with echocardiographically documented hypertrophic cardiomyopathy. Emergency coronary arteriography revealed embolic occlusion of the proximal left circumflex coronary artery and the first diagonal branch. Intracoronary thrombolysis with urokinase and subsequent balloon angioplasty was successful. Transesophageal echocardiography revealed thrombus in the left atrial appendage. Coronary arteriography performed on the 46th hospital day revealed a patent left circumflex coronary artery and diagonal branch. The patient was discharged uneventfully.
\end{abstract}

(Internal Medicine 36: 613-617, 1997)

Key words: left atrial appendage thrombus, transesophageal echocardiography, atrial fibrillation

\section{Introduction}

There have been several reports of patients with hypertrophic cardiomyopathy and normal coronary arteries who have suffered acute myocardial infarction, but none of them have been angiographically documented to have coronary embolism (1-10). Here, we report a case of hypertrophic cardiomyopathy complicated with acute myocardial infarction due to coronary embolism. Successful coronary revascularization allowed the patient to achieve a good recovery.

\section{Case Report}

A 75-year-old man had echocardiographically documented hypertrophic cardiomyopathy (HCM) and was being treated with verapamil ( $160 \mathrm{mg}$ daily) to control the ventricular rate. He was admitted to our hospital in October 1996 with chest discomfort lasting for three hours. He had suffered a cerebral infarction in January 1986. At that time, electrocardiography revealed atrial fibrillation and echocardiographic findings were compatible with hypertrophic non-obstructive cardiomyopathy. His cerebral infarction was thought to be due to embolism, so warfarin potassium was prescribed and the prothrombin time was maintained at an international normalized ratio (INR) of 2.1. There was no history of hypertension, diabetes mellitus, or dyslipidemia, but he had smoked half a pack of cigarettes daily for 50 years.

On admission, he was pale with cold perspiration and cyanotic. His pulse was irregular with a rate of $90 / \mathrm{min}$, and blood pressure was $90 / 60 \mathrm{mmHg}$. Physical examination revealed bilateral inspiratory fine crepitations, a laterally displaced apical impulse, and Levine II/VI systolic ejection murmur at the 4th left sternal border. The chest X-ray film demonstrated pulmonary edema and marked cardiomegaly at a cardiothoracic ratio (CTR) of $71 \%$.

Electrocardiography revealed atrial fibrillation accompanied by ST segment elevation in leads I, II, $\mathrm{V}_{\mathrm{L}},{ }_{\mathrm{a}} \mathrm{V}_{\mathrm{F}}$, and $\mathrm{V}_{4}$ $\mathrm{V}_{6}$, as well as $T$ wave inversion in leads $\mathrm{V}_{3}-\mathrm{V}_{5}$ (Fig. 1B). Right heart catheterization showed a pulmonary artery pressure of $72 /$ $40 \mathrm{mmHg}$ and mean pulmonary artery wedge pressure of 42 mmHg. An intra-aortic balloon pump (IABP) was inserted. Coronary arteriography revealed total occlusion of the proximal left circumflex coronary artery and the mid-portion of the first diagonal branch (Fig. 2A, B). The right coronary artery was normal. Although intracoronary thrombolysis with 480,000 units of urokinase moved both thrombi slightly distal to the occluded portion, reperfusion was not obtained. There was no significant stenosis at the occluded segments. Subsequently, balloon angioplasty was performed for the left circumflex artery (Fig. 2C, D). However, delayed flow of contrast medium to the posterolateral branch of the circumflex artery and the first diagonal branch was still noted (Fig. 2E). The serum creatine kinase concentration peaked at $6,550 \mathrm{IU} / l$. Although the IABP was removed on the 3rd hospital day, olprinone hydrochloride was administered intravenously for 30 days because of intractable congestive heart failure. Transthoracic echocardiography

From the Department of Internal Medicine, Tokyo Metropolitan Bokutou Hospital, Tokyo

Received for publication January 7, 1997; Accepted for publication May 30, 1997

Reprint requests should be addressed to Dr. Toru Iwama, the Department of Internal Medicine, Tokyo Metropolitan Bokutou Hospital, 4-23-15 Koutoubashi, Sumida-ku, Tokyo 130 


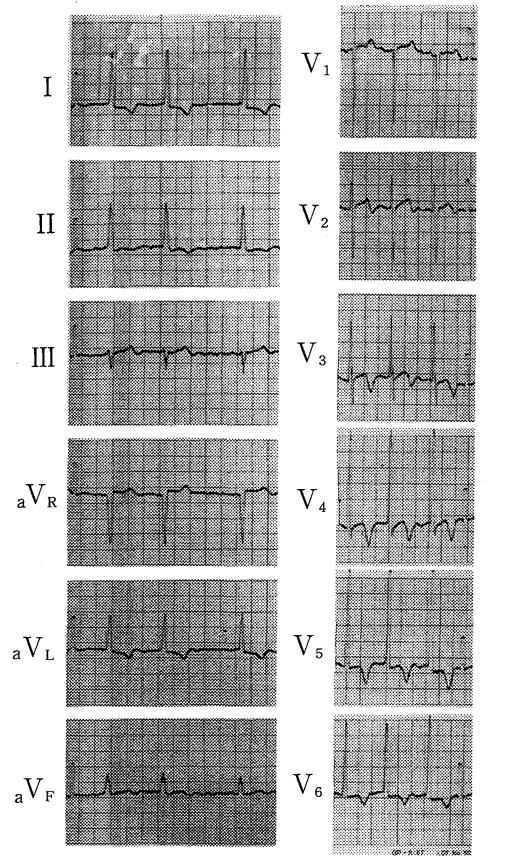

A

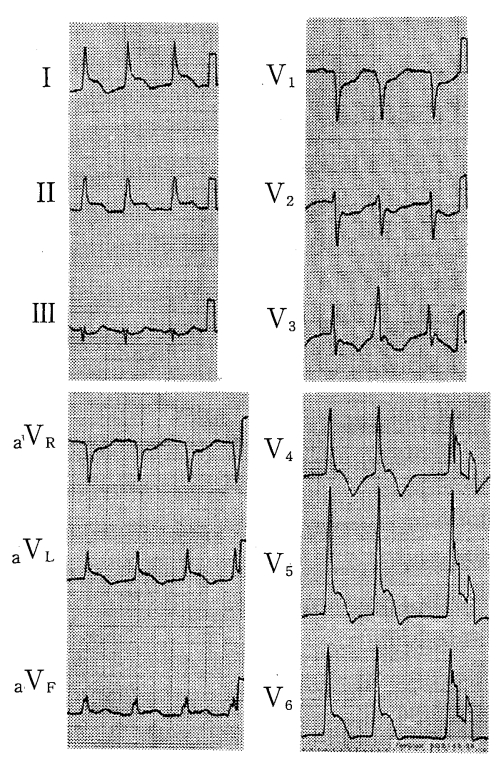

B

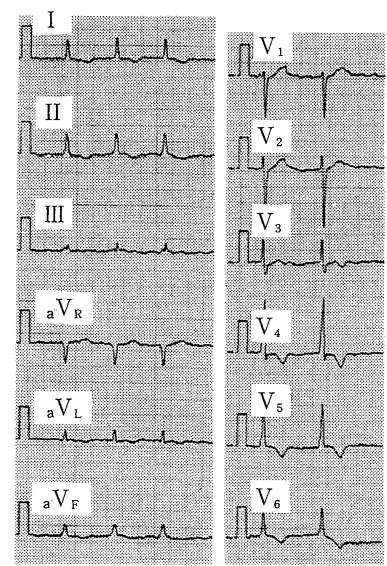

C

Figure 1. Electrocardiograms. A) Eighteen months before admission, revealing atrial fibrillation and $T$ wave inversion in leads $I,{ }_{a} V_{L}$, and $V_{3}-V_{6}$. B) On admission, revealing ST elevation in leads $I, I_{,}, V_{L},{ }_{a} V_{F}$, and $V_{4}-V_{6} . C$ ) On the 36th hospital day, revealing low voltages in all leads and no $Q$ waves.
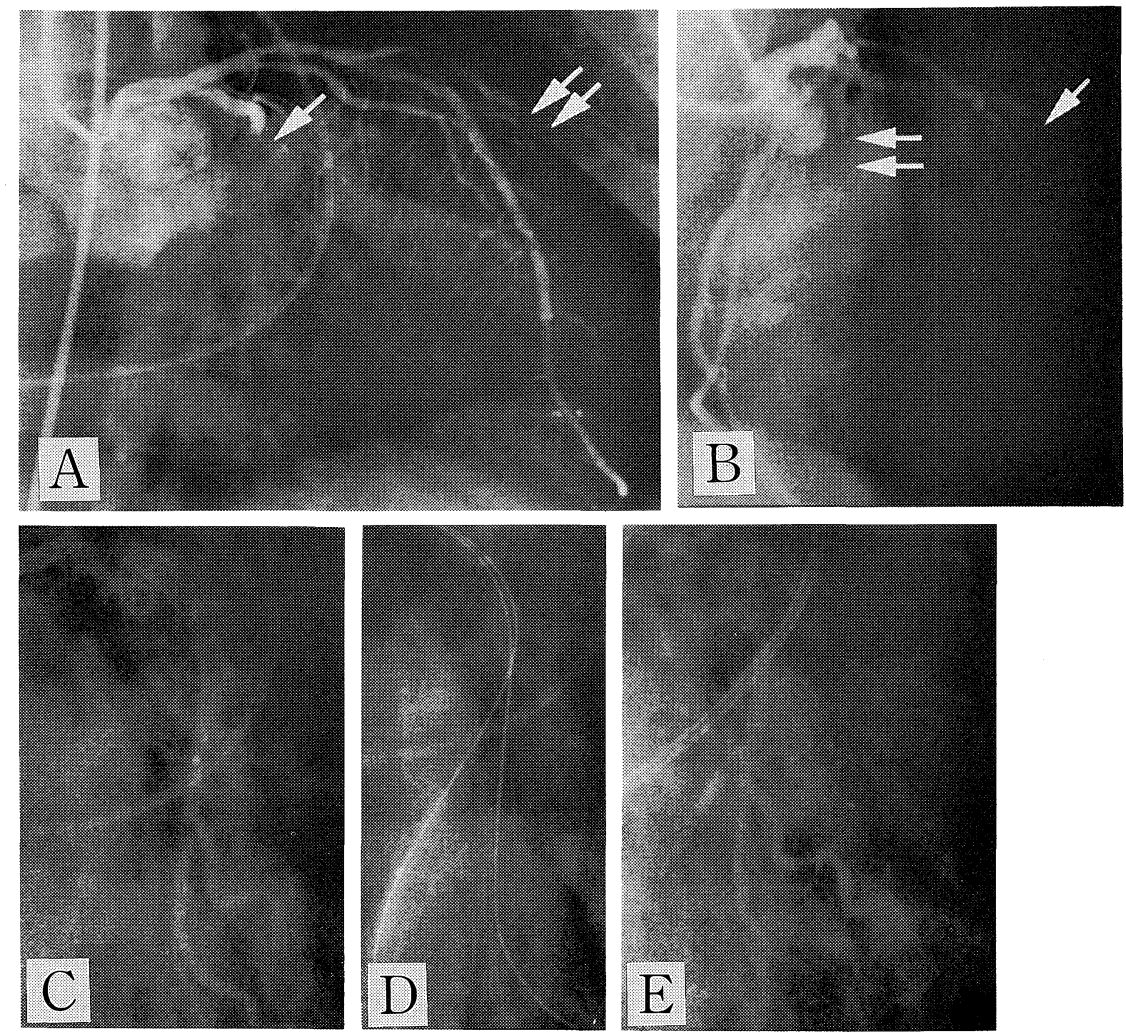

Figure 2. Coronary arteriography on admission. A) RAO view. B) LAO cranial view. The proximal left circumflex coronary artery (arrow) and the mid portion of the first diagonal branch (double arrow) are occluded. C)-E) Angioplasty of the left circumflex artery. LAO: left anterior oblique, RAO: right anterior oblique. 



Figure 3. Transesophageal echocardiography on the 42nd hospital day. There is asymmetric ventricular septal hypertrophy (arrow) as well as thrombus (double arrow) in the left atrial appendage. LA: left atrium, LAA: left atrial appendage, $L V$ : left ventricle, $R V$ : right ventricle.
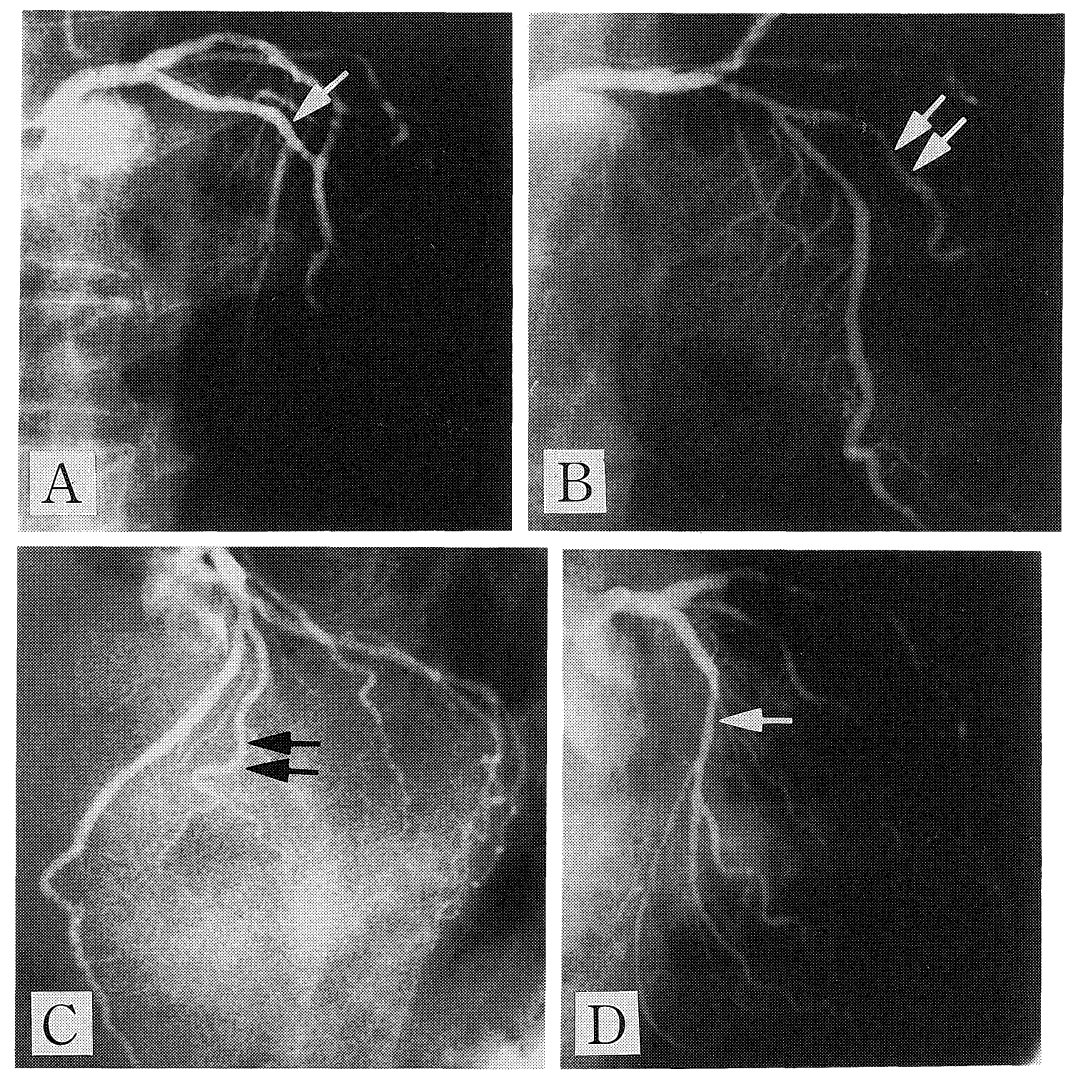

Figure 4. Coronary arteriography on the 46th hospital day. A) RAO view. B) AP cranial view. C) LAO cranial view. D) RAO caudal view. Except for an $80 \%$ stenosis of the posterolateral branch of the left circumflex coronary artery, no significant lesions are seen. Arrow and double arrow indicate regions occluded on admission. AP: antero-posterior, LAO: left anterior oblique, RAO: right anterior oblique. 

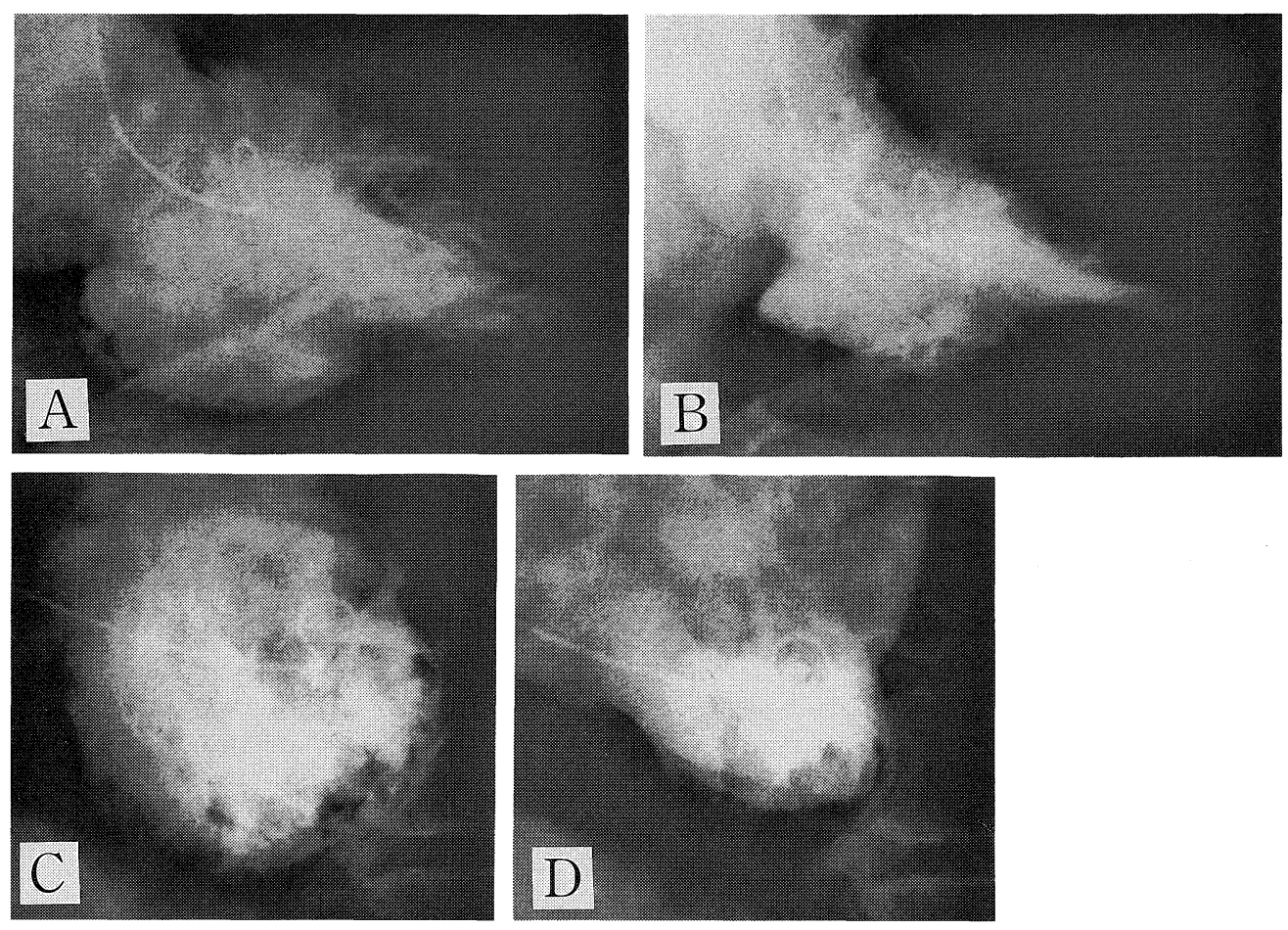

Figure 5. Left ventriculography on the 46th hospital day. A) RAO view in diastole. B) RAO view in systole. C) LAO view in diastole. D) LAO view in systole. Hypokinesia of the infero-posterior region is seen. LAO: left anterior oblique, RAO: right anterior oblique.

performed on the 42nd hospital day showed Maron's type III hypertrophic cardiomyopathy with interventricular septal/left ventricular posterior wall thickness of $16 / 8 \mathrm{~mm}$, hypokinesia of inferior and posterior segments, mildly enlarged left atrium with a diameter of $37 \mathrm{~mm}$, and mild mitral regurgitation. There was no significant systolic anterior movement of the mitral valve. Transesophageal echocardiography performed on the same day showed a high-echogenic, clear-bordered structure measuring $18 \times 8 \mathrm{~mm}$ with independent mobility in the left atrial appendage (Fig. 3A, B). No smoke-like echo was seen in the left atrium. Electrocardiography on the 36th hospital day showed lower voltages in all leads compared with before admission and no abnormal Q waves (Fig. 1A, C). The patient gradually recovered and cardiac catheterization was repeated on the 46th hospital day, with complete recanalization of the left circumflex coronary artery and the first diagonal branch (Fig. 4A, B, C). However there was $80 \%$ stenosis of the posterolateral branch of the left circumflex artery, that was thought to be due to an intimal tear during balloon inflation (Fig. 4D). Left ventriculography showed moderate dysfunction with infero-posterior segment hypokinesia (Fig. 5). Warfarin potassium was administered with the prothrombin time maintained at an INR of 2.2 and the patient was discharged.

\section{Discussion}

Although there have been several reports of HCM patients with coronary arteries angiographically demonstrated to be normal who developed acute myocardial infarction (1-9), only one had evidence of a single coronary thrombus (8). Maron et al (10) proposed the following as possible causes of myocardial infarction in patients who had HCM without significant coronary atherosclerosis: 1) insufficient blood supply relative to the increased cardiac muscle mass, 2) coronary embolism, 3) narrowing of the intramural coronary arteries, 4) coronary spasm, and 5) brief periods of hypoxia or hypotension. As the present patient had chronic atrial fibrillation, thrombus in the left atrial appendage, and two thrombotic coronary occlusions, the etiology of infarction in this case was apparently coronary embolism. Therefore, this is the first case report of HCM complicated with acute myocardial infarction due to coronary embolism. It should also be mentioned that transesophageal echocardiography was useful for detecting left atrial appendage thrombus (11).

Coronary embolism is generally considered to be a rare cause of acute myocardial infarction, with less than 150 cases reported in the literature since Virchow's original description in 1856 (12). However, in a study of 419 autopsy cases of myocardial infarction by Prizel et al (13), 55 patients (13\%) showed evidence of coronary embolism, suggesting that it was not rare. Although the most common origin of coronary emboli has been identified as bacterial vegetations in patients with infective endocarditis (14), intracardiac thrombosis is being identified as the cause in an increasing number of cases (15). 


\section{HCM with AMI due to Coronary Embolism}

The conditions predisposing to coronary embolism include valvular heart disease (40\%), cardiomyopathy (29\%), coronary atherosclerosis (16\%), and chronic atrial fibrillation (24\%) (13). The main site of embolization is the left anterior descending artery (56-68\%), followed by the right coronary (12-33\%), left circumflex artery (6.8-24\%), and left main coronary trunk $(10 \%)$. If the emboli are multiple, the site of coronary occlusion may be difficult to diagnose because typical ischemic changes are not demonstrated by electrocardiography (16). In the present case, electrocardiography showed evidence of acute high lateral and infero-lateral myocardial infarction which was very unlikely to have been caused by single-vessel occlusion.

\section{References}

1) Newman PE. Acute myocardial infarction with angiographically demonstrated normal coronary arteries in the presence of hypertrophic cardiomyopathy. Chest 78: 893, 1980.

2) Karibe T, Kouda $Y$, Satou H, et al. A case of hypertrophic cardiomyopathy with acute myocardial infarction induced by paroxysmal supraventricular tachycardia. Jpn Circ J 50: Suppl I: 83, 1986 (Abstract in Japanese).

3) Iwase T, Hoshino T, Yokoyama S, et al. A case of hypertrophic cardiomyopathy complicated with hypertension and acute myocardial infarction. Jpn Circ J 51: Suppl II: 297, 1987 (Abstract in Japanese).

4) Andoh M, Sone T, Kawaguchi K, et al. A case of hypertrophic cardiomyopathy with acute septal infarction induced by paroxysmal atrial fibrillation. Shinzou 21: 1230, 1989 (in Japanese).

5) Komiyama N, Nishiyama S, Nakanishi S, et al. Clinical diagnosis and pathogenesis of myocardial infarction complicated by hypertrophic cardiomyopathy: Review of eight cases. J Cardiol 19: 805, 1989 (in Japanese, with English abstract).

6) Mukaida H, Mizunuma $\mathrm{Y}$, Tashiro A, et al. A case of acute myocardial infarction with angiographically demonstrated normal coronary arteries in the presence of hypertrophic cardiomyopathy. Jpn Circ J 53: Suppl I: 49, 1989 (Abstract in Japanese).

7) Miyake S, Kawara T, Uesaka H, et al. Hypertrophic cardiomyopathy complicated by acute myocardial infarction. Nippon Naika Gakkai Zasshi 76: 154, 1987 (Abstract in Japanese).

8) Hirokawa J, Horimoto M, Igarashi $\mathrm{K}$, et al. Hypertrophic cardiomyopathy with normal coronary arteries complicated by acute myocardial infarction due to intracoronary thrombus. Shinzou 23: 1273, 1991 (in Japanese).

9) Yoshimura R, Akahoshi R, Sugiura T, et al. Silent myocardial infarction in the absence of significant atherosclerosis in hypertrophic cardiomyopathy. Iryo 47: 211, 1993 (in Japanese with English abstract).

10) Maron BJ, Epstein SE, Roberts WC. Hypertrophic cardiomyopathy and transmural myocardial infarction without significant atherosclerosis of the extramural coronary arteries. Am J Cardiol 43: 1086, 1979.

11) ELAT Study Group. ELAT study (embolism in left atrial thrombi): baseline clinical and echocardiographic data. Cardiology 86: 457, 1995.

12) Virchow R. Ueber capillare embolie. Pathol Anat 9: 307, 1856.

13) Prizel KR, Hutchins GM, Bulkley BH. Coronary artery embolism and myocardial infarction. Ann Intern Med 88: 155, 1978.

14) Wenger NK. Coronary artery embolism. Review of the literature and presentation of fifteen cases. Am J Med 21: 549, 1958.

15) Loire R, Tabib A. Coronary embolism. Apropos 61 anatomo-clinical cases. Arch Mal Coeur Vaiss 78: 821, 1985 (in French with English abstract).

16) Maddoux GL, Goss JE, Ramo BW, et al. Left main coronary artery embolism: a case report. Cath Cardiovasc Diagn 13: 394, 1987. 